\title{
Interstellar and interplanetary solids in the laboratory
}

\author{
Emmanuel Dartois ${ }^{1} \dagger$, Ivan Alata ${ }^{3}$, Cécile Engrand ${ }^{2}$, Rosario \\ Brunetto $^{1}$, Jean Duprat ${ }^{2}$, Thomas Pino ${ }^{3}$, Eric Quirico ${ }^{4}$, Laurent \\ Remusat $^{5}$, Noémie Bardin ${ }^{2}$, Smail Mostefaoui ${ }^{5}$, Gilles Morinaud ${ }^{1}$, \\ Bruno Crane $^{1}$, Nicolas Szwec ${ }^{1}$, Lucie Delauche ${ }^{2}$, Frédéric Jamme ${ }^{6}$, \\ Christophe Sandt ${ }^{6}$ and Paul Dumas ${ }^{6}$ \\ ${ }^{1}$ Institut d'Astrophysique Spatiale, UMR8617 CNRS-Univ. Paris-Sud, Université Paris-Saclay, \\ Bât. 121, Univ. Paris-Sud, 91405 Orsay, France \\ ${ }^{2}$ Centre de Sciences Nucléaires et de Sciences de la Matière, UMR8609 CNRS/IN2P3-Univ. \\ Paris-Sud, Université Paris-Saclay, Bât. 104, 91405 Orsay, France \\ ${ }^{3}$ ISMO, UMR 8214-CNRS Université Paris Sud, bât 210, 91405 Orsay Cedex, France \\ ${ }^{4}$ IPAG, UJF-Grenoble 1/CNRS-INSU, UMR 5274, 38041 Grenoble, France \\ ${ }^{5}$ IMPMC, UMR 7590 CNRS, UPMC, IRD, MNHN, 61 rue Buffon, 75005 Paris, France \\ ${ }^{6}$ Synchrotron SOLEIL, BP48, Saint Aubin, 91192 Gif sur Yvette, France
}

\begin{abstract}
The composition of interstellar matter is driven by environmental parameters and results from extreme interstellar medium physico-chemical conditions. Astrochemists must rely on remote observations to monitor and analyze the interstellar solids composition. They bring additional information from the study of analogues produced in the laboratory, placed in simulated space environments. Planetologists and cosmochemists access and spectroscopically examine collected extraterrestrial material in the laboratory. Diffuse interstellar medium and molecular clouds observations set constraints on the composition of organic solids that can then be compared with collected extraterrestrial materials analyses, to shed light on their possible links.
\end{abstract}

Keywords. ISM: dust, extinction, ISM: lines and bands, interplanetary medium, comets: general, Kuiper Belt, Oort Cloud

\section{Introduction}

Carbonaceous matter in the ISM. The interstellar medium is populated by different forms of carbonaceous solids: polyaromatic hydrocarbons (giving rise to emission bands, notably at $3.3,6.2,7.7,8.6$, and 11.3 microns, the AIBs); amorphous carbons (difficult to observe remotely, as no specific features are associated with them, injected e.g. by the so-called late-type carbon stars); hydrogenated amorphous carbons (a-C:H, observed at $3.4 \mu \mathrm{m}$ in absorption against background infrared sources, with an ubiquitous presence in the diffuse interstellar medium -DISM - of galaxies), (nano-)diamonds (observed via specific infrared emission bands at 3.43 and 3.53 microns around three young stars); fullerenes (observed via mid-infrared -17.4 and $18.9 \mu \mathrm{m}$ - then $-7.04 \mu \mathrm{m}$ - emission bands for $\mathrm{C}_{60}$, and recently confirmed for some absorption in the near infrared, in the laboratory, for the ionized form $\mathrm{C}_{60}^{+}$; Campbell et al. 2015); and ice mantles in dense regions, that may be precursors for organic residues. The detections and spectacular spectral attributions for some of the carriers (e.g. nano-diamonds, $\mathrm{C}_{60}^{+}$), essential to constrain the physical and chemical environment of astrophysical media, are to be balanced by the carbonaceous material global abundances when dealing with their incorporation into what will constitute the main interplanetary organic matter content, in objects such as

$\dagger$ emmanuel.dartois@ias.u-psud.fr, to whom correspondence should be addressed 
protoplanetary disks. In term of abundance, the a-C:H dust component, on which we focus hereafter, constitues an important carbon reservoir, at least as important as the AIB carrier one, and to which must be eventually added the organic residues reservoir in dense regions evolution.

\section{2. a-C:H from ISM observations to analogues and meteorites}

a-C:H evolution. a-C:H are an important component of the C-rich phases in the Galaxy and in the recent decades, they have been observed in a rising number of extragalatic lines of sights. The understanding of the structural unit of this material mixing aliphatic and aromatic components has evolved since its observation in other galaxies ISM, with less circumstellar contamination of the spectra at such larger scale (e.g. Dartois et al. 2007). Such hydrogenated amorphous carbons analogues can be produced in the laboratory and submitted to VUV irradiations, and the destruction cross-section of $\mathrm{CH}$ bonding being monitored with the $3.4 \mu \mathrm{m}$ band (e.g. Mennella et al. 2001, Alata et al. 2014a). The products of this VUV photolytic destruction can also be monitored. The main product is $\mathrm{H}_{2}$ and the photolytic $\mathrm{H}_{2}$ formation rate can be estimated (Alata et al. 2014a). Other photoproducts are monitored, including $\mathrm{CH}_{4}$ as well as hydrocarbons of higher masses (Alata et al. 2014b). Such photolytic mechanisms are of interest in the ISM, but also for interface regions involving molecular clouds illuminated by powerful UV sources, known as Photon Dominated Regions (PDR), with $\mathrm{H}_{2}$ formation observed at high grain temperature, and small hydrocarbons abundance increase at the exposed cloud interface.

From laboratory experiments, a VUV $\mathrm{H}_{2}$ formation rate coefficient of up to 1.25 $2.5 \times 10^{-16} \mathrm{~cm}^{3} \mathrm{~s}^{-1}$ is estimated, assuming that $5-10 \%$ of the cosmic carbon is locked into the a-C:H dust component. This rate can be compared to observational estimates of $0.3-1.5 \times 10^{-16} \mathrm{~cm}^{3} \mathrm{~s}^{-1}$ in some PDRs (Habart et al. 2004), in regions where surface reactions involving physisorption of $\mathrm{H}$ atoms would fail to produce $\mathrm{H}_{2}$ due to the high dust grain temperature. Thus VUV irradiation implies efficient formation rates at low to high grains temperatures, and contributes to the formation of species whose abundance is observed enhanced in these regions.

Incorporation of a-C:H within solar system matter? Early comparisons between extraterrestrial carbonaceous matter extracted from primitive meteorites, that consists of a mixture of insoluble and soluble organic matter (IOM and SOM) were performed via infrared spectroscopy on the IOM fraction (e.g. Ehrenfreund et al. 1991). Limited to the $3.4 \mu \mathrm{m}$ spectral window, the relative good spectral match suggested that the observed interstellar matter was similar to the meteorite organic extract. The extension to the mid infrared regions, thanks to the satellites observations outside of the atmosphere shows that the spectroscopic comparison with the IOM in the 5 to $10 \mu \mathrm{m}$ is no longer comparable to the ISM hydrogenated carbons contributing at $3.4 \mu \mathrm{m}$. The IOM structure in the Orgueil meteorite and in the labile fraction in CM chondrites have definitely another chemical network (Kitajima et al. 2002, Remusat et al. 2007, Derenne \& Robert 2010). Several mid-infrared absorptions (Kebukawa et al. 2011, Orthous-Daunay et al. 2013) for the IOM do not allow to provide a correct spectral match, and can be related to the presence of higher amounts of oxygen heterotaoms in the carbonaceous network. In the ISM a-C:H dust, there are no evidence for a large amount of incorporation of such oxygen heteroatoms, nor nitrogen ones. This does not precludes the ISM dust being a precursor modified during the meteorites formation process. What are therefore the observed evidence of the solids evolution from the diffuse to dense medium. The DISM a-C:H signatures disappear at the interface of molecular clouds. In the laboratory, a$\mathrm{C}: \mathrm{H}$ analogues have been shown to potentially dehydrogenate under the conditions met 
in the dense phase and/or at the interface between diffuse and dense clouds (Mennella et al. 2003, Godard et al. 2011), thus, before being incorporated into a protosolar nebula. Deeper, the conditions are met for interstellar ice mantles growth. They may, upon irradiation, lead to an organic residue precursor material, provided that the subsequent evolutionary pathways can lead to some kind of IOM.

A location question also arises when dealing with the comparison between meteorites IOM and ISM materials. A large amount of comparisons between extraterrestrial collected organic matter and the interstellar medium dust observations have been focusing on meteorites arising from the asteroid belt region, because of the possibility to extract the IOM from these meteorites. In the recent decades, an effort is pushed onto the characterisation of organic material from cosmic dust collected on Earth, such as interplanetary dust particles (IDPs) and micrometeorites. Among these dust particles, some IDPs are traveling from the outer Solar System, and their composition are detailed in other contributions of this focus meeting. We will focus hereafter on the organic component of some rare specific ultra carbonaceous Antarctic micrometeorites (UCAMMs). These UCAMMs were discovered as a small fraction in at least two Antarctic micrometeorites collections, such as those reported from the Dome Fuji collection (Nakamura et al. 2005), and also present in the Concordia collection of the CSNSM, from the French Italian Dome C station (Duprat et al. 2007, 2010).

Combined with NanoSIMS measurements, scanning electron micrographs and X-ray maps measurements of two of these UCAMMs showed that they are very rich in organic content (more than 50\% and up to $95 \%$ of the whole UCAMM atoms being organic matter), making them as n̈atural IOMmicrometeorites (Dartois et al. 2013). They can be studied whithout the need for chemicals to extract their organic content. They possess a high deuterium enrichment, even when compared to the $\mathrm{D} / \mathrm{H}$ recorded in cometary organic molecules remote observations (Bockelée-Morvan et al. 2015), and often associated with a formation in cold environment such as the external regions of the young protoplanetary disk. Their organic matter presents infrared (Dartois et al. 2013) and Raman spectra (Dobrica et al. 2011) similar to laboratory analogues of polyaromatic hydrogenated carbon nitrides, and display nitrogen concentrations characterized by bulk atomic N/C ratios of 0.05 and 0.12 (locally exceeding 0.15 ). Such nitrogen-rich solids have not been observed up to now in the interstellar medium. In the solar system, such N-rich materials, are seldom encountered. In addition, experimentally, the inclusion of such high amounts of nitrogen (10-20\%) in a (oxygen poor) carbon nitride requires a carbon rich precursor, and energetic processes in an N-dominated environment (i.e. $\mathrm{H}_{2} \mathrm{O}$ ice depleted when dealing with ices).

The UV photolysis or cosmic ray radiolysis of $\mathrm{N}$ - and C-rich ices may lead to these nitrogen-rich carbonaceous materials. Beyond the trans-Neptunian region, in the cold outer regions of our solar system, the surface of small icy bodies can meet these conditions, and fix isotopic local enrichment in a cold chemistry context. Even closer to us, and for large bodies such as Pluto, $\mathrm{N}_{2}$ and $\mathrm{CH}_{4}$-rich ice surfaces are observed (e.g. Douté et al. 1999, Protopapa et al. 2008, Grundy et al. 2013).

The UCAMMs provide a unique collected extraterrestrial material to get potentially insight into the evolution of matter incorporated from the protosolar nebula into the solar system disk. Comparisons and differences between UCAMMs and many of the solar system collected materials and ISM spectra are provided in a summary figure in a similar review in Dartois et al. (2014a). UCAMMs would result from the physicochemical processes that occurred beyond a nitrogen snow-line, revealing organic material from the outer regions of the Solar System. The remote observations currently possible 
cannot investigate surface reflectance spectra for the many small icy bodies at such distances.

\section{Implications}

The IOM extracted from asteroid belt meteorites and observed interstellar a-C:H in absorption show differences in their infrared spectral signatures. Ultracarbonaceous Antarctic micrometeorites (UCAMMs), whose isotopic signatures suggest an origin in the cold outer regions of the solar system, contain a bulk hydrogenated carbon nitride organic matter, with an $\mathrm{N}$ content well above that of the IOM extracted from asteroid belt meteorites and many IDPs. This overall picture does not suggest any evidence of ISM materials directly imported and incorporated at large scale in the solar system. If specific presolar grains (e.g. highly refractory $\mathrm{SiC}$, graphite) are whithout doubt tracing an heritage from interstellar matter in meteorites, the bulk of the organic matter seems to be mainly probing physicochemical mechanisms occurring in the young protoplanetary solar system. To decipher the fate of organic matter in such environments, laboratory studies are essentiel to simulate the future of materials observed in the ISM cycle.

\section{References}

Alata, I., Cruz-Diaz, G. A., Muñoz Caro, G. M., \& Dartois, E. 2014a, A\&AA, 569, A119

Alata, I., Jallat, A., Gavilan, L., Chabot, M., Cruz-Diaz, G. A., Muñoz Caro, G. M., Béroff, K., \& Dartois, E. 2014b, A\&BA, 569, A119

Bockelée-Morvan et al. 2015, Spac. Sci. Rev, 1.

Campbell, E. K., Holz, M., Gerlich, D., \& Maier, J. P. 2015, Nature 523, 322

Dartois, E., Geballe, T. R., Pino, T., Cao, A.-T., Jones, A., Deboffle, D., Guerrini, V., Bréchignac, P., \& D'Hendecourt, L. 2007, A\&SA, 463, 635-640.

Dartois, E., Engrand, C., Brunetto, R., Duprat, J., Pino, T., Quirico, E., Remusat, L., Bardin, N., Briani, G., Mostefaoui, S., Morinaud, G., Crane, B., Szwec, N., Delauche, L., Jamme, F., Sandt, Ch., \& Dumas, P., 2013, Icarus, 224, 243

Derenne, S. \& Robert, F., 2010, Meteorit. Planet. Sci. 45, 1461

Dobrica, E., Engrand, C., Quirico, E., Montagnac, G., \& Duprat, J. 2011, Meteoritics and Planetary Science, 46, 1363

Douté, S., Schmitt, B., Quirico, E., Owen, T. C., Cruikshank, D. P., de Bergh, C., Geballe, T. R., \& Roush, T. L., 1999, Icarus 142, 421-444.

Duprat, J., Engrand, C., Maurette, M., Kurat, G., Gounelle, M., \& Hammer, C., 2007, Adv. Space Res., 39, 605

Duprat, J., Dobrică, E., Engrand, C., et al. 2010, Science, 328, 742

Ehrenfreund, P., Robert, F., D'Hendecourt, L., \& Behar, F., 1991, A\&A 252, 712

Godard, M., Féraud, G., Chabot, M., Carpentier, Y., Pino, T., Brunetto, R., Duprat, J., Engrand, C., Bréchignac, P., D'Hendecourt, L., \& Dartois, E., 2011, A\& A, 529, A146.

Grundy, W. M., Olkin, C. B., Young, L. A., Buie, M. W., \& Young, E. F., 2013, Icarus 223, 710

Kitajima, F., Nakamura, T., Takaoka, N., \& Murae, T., 2002, Geochim. Cosmochim. Acta, 66, 163

Kebukawa, Y., Alexander, C. M. O.'D., \& Cody, G. D., 2011, Geochim. Cosmochim. Acta, 75, 3530

Mennella, V., Muñoz Caro, G. M., Ruiterkamp, R., et al. 2001, A $\& A$ 367, 355

Mennella, V., Baratta, G. A., Esposito, A., Ferini, G., \& Pendleton, Y. J., 2003, ApJ 587, 727

Nakamura, T., Noguchi, T., Ozono, Y., Osawa, T., \& Nagao, K., 2005, Meteorit. Planet. Sci. Suppl., 40, 5046.

Orthous-Daunay, F.-R., Quirico, E., Beck, P., Brissaud, O., Dartois, E., Pino, T., \& Schmitt, B., 2013, Icarus, 223, 534

Protopapa, S., Boehnhardt, H., Herbst, T. M., Cruikshank, D. P., Grundy, W. M., Merlin, F., \& Olkin, C. B., 2008, A\&\&A 490, 365

Remusat, L., Robert, F., \& Derenne, S., 2007, Comptes Rendus Geoscience, 339, 895 